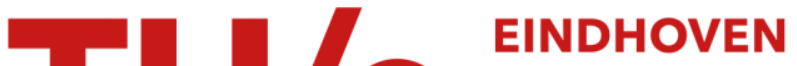 UNIVERSITY OF TECHNOLOGY
}

\section{Effect of polaron diffusion on exciton-polaron quenching in disordered organic semiconductors}

\section{Citation for published version (APA):}

Coehoorn, R., Zhang, L., Bobbert, P. A., \& van Eersel, H. (2017). Effect of polaron diffusion on exciton-polaron quenching in disordered organic semiconductors. Physical Review B, 95(13), [134202].

https://doi.org/10.1103/PhysRevB.95.134202

DOI:

10.1103/PhysRevB.95.134202

Document status and date:

Published: 06/04/2017

\section{Document Version:}

Publisher's PDF, also known as Version of Record (includes final page, issue and volume numbers)

\section{Please check the document version of this publication:}

- A submitted manuscript is the version of the article upon submission and before peer-review. There can be important differences between the submitted version and the official published version of record. People interested in the research are advised to contact the author for the final version of the publication, or visit the $\mathrm{DOI}$ to the publisher's website.

- The final author version and the galley proof are versions of the publication after peer review.

- The final published version features the final layout of the paper including the volume, issue and page numbers.

Link to publication

\section{General rights}

Copyright and moral rights for the publications made accessible in the public portal are retained by the authors and/or other copyright owners and it is a condition of accessing publications that users recognise and abide by the legal requirements associated with these rights.

- Users may download and print one copy of any publication from the public portal for the purpose of private study or research.

- You may not further distribute the material or use it for any profit-making activity or commercial gain

- You may freely distribute the URL identifying the publication in the public portal.

If the publication is distributed under the terms of Article 25fa of the Dutch Copyright Act, indicated by the "Taverne" license above, please follow below link for the End User Agreement:

www.tue.nl/taverne

Take down policy

If you believe that this document breaches copyright please contact us at:

openaccess@tue.nl

providing details and we will investigate your claim. 


\title{
Effect of polaron diffusion on exciton-polaron quenching in disordered organic semiconductors
}

\author{
R. Coehoorn, ${ }^{*}$ L. Zhang, and P. A. Bobbert \\ Department of Applied Physics and Institute for Complex Molecular Systems, Eindhoven University of Technology, \\ P.O. Box 513, NL-5600 MB Eindhoven, The Netherlands \\ H. van Eersel \\ Simbeyond B.V., P.O. Box 513, NL-5600 MB Eindhoven, The Netherlands
}

(Received 6 February 2017; published 6 April 2017)

\begin{abstract}
Exciton-polaron quenching (EPQ) is a major efficiency loss process in organic optoelectronic devices, in particular at high excitation densities. Within commonly used models, the rate is assumed to be given by the product of the exciton density, the polaron density, and a constant EPQ rate coefficient, which is proportional to the polaron diffusion coefficient and an EPQ capture radius. In this work, we study the effects of polaron diffusion on the EPQ rate in energetically disordered materials with a Gaussian density of states using kinetic Monte Carlo simulations, and show that the effective rate coefficient can depend strongly on the polaron concentration and on the electric field. We furthermore find that under realistic conditions, the effective value of the capture radius can exceed the expected value of $\sim 1 \mathrm{~nm}$ by up to two orders of magnitude. To a first approximation, the simulation results can be understood from macroscopic diffusion theory, adapted at finite electric fields to include the observed "polaron wind" effect. However, for strongly disordered systems we find distinct deviations from that theory, related to the very small time and spatial scales involved in the capture process.
\end{abstract}

DOI: 10.1103/PhysRevB.95.134202

\section{INTRODUCTION}

At high excitation densities, exciton-polaron-quenching (EPQ) processes can give rise to a loss of the internal quantum efficiency of organic optoelectronic devices such as organic light-emitting diodes (OLEDs), organic photovoltaic devices under concentrated solar illumination, organic lightemitting field-effect transistors, and organic lasers. Upon such a process, the energy of an exciton on a donor molecule is first transferred to a positively or negatively charged polaron on an acceptor molecule, so that the donor molecule returns to the ground state. Subsequently, the excess energy on the acceptor molecule is lost rapidly via vibrational relaxation processes. Alternative terminologies used in the literature to indicate such bimolecular loss processes are "excitonpolaron annihilation" and "exciton-charge annihilation". For phosphorescent OLEDs, within which the emission originates from relatively long-lived excitons with predominantly triplet character, triplet-polaron quenching (TPQ) can contribute significantly to the efficiency rolloff at high luminance levels [1]. For donor-acceptor blends with nanomorphologies which are optimal for photovoltaic operation, exciton-polaronquenching reactions have also been found to be a relevant loss process [2-4].

Experimental methods for studying EPQ include transient absorption spectroscopy [2,5] of thin-film materials, within which the polarons are produced as a result of exciton dissociation, combined photoluminescence (PL) and charge carrier transport measurements on organic thin-film transistors [6], and transient and steady-state photoluminescence studies of unipolar (single-carrier) metal/semiconductor/metal sandwich-type devices [7-9]. In the latter devices, the application of a voltage difference between the electrode layers

\footnotetext{
*r.coehoorn@tue.nl
}

gives rise to an injected polaron density. The time- $(t-)$ dependent exciton-polaron-quenching rate $r_{\mathrm{EPQ}}(t)$ is conventionally expressed in terms of an exciton-polaron-quenching rate coefficient $k_{\mathrm{EPQ}}(t)$, using the expression $r_{\mathrm{EPQ}}(t) \equiv k_{\mathrm{EPQ}}(t) n_{p}$, with $n_{p}$ the polaron volume density. The decrease of the exciton volume density $n_{E}$ due to EPQ is then given by

$$
\left.\frac{d n_{E}(t)}{d t}\right|_{\mathrm{EPQ}}=-k_{\mathrm{EPQ}}(t) n_{p} n_{E}(t) .
$$

In general, a sum of contributions due to quenching at electrons and holes should be considered. Experimental values obtained for $k_{\mathrm{EPQ}}$ range from typically $(0.01-1) \times 10^{-18} \mathrm{~m}^{3} / \mathrm{s}$ for TPQ in phosphorescent small-molecule host-guest systems used in OLEDs [7-13] to values which can be three orders of magnitude larger for singlet-polaron quenching (SPQ) in fluorescent polymer materials [5].

From macroscopic diffusion theory [14-17], the EPQ rate coefficient is expected to be given by

$$
k_{\mathrm{EPQ}}(t)=4 \pi D R_{c}\left(1+\frac{R_{c}}{\sqrt{\pi D t}}\right),
$$

with $D$ the mutual diffusion coefficient, i.e., the sum of the exciton and polaron diffusion coefficients. $R_{c}$ is an effective exciton-polaron capture radius, and $t$ is the time which has elapsed since the moment of adding the exciton to the system. The first term is due to long-range three-dimensional diffusion [see Fig. 1(a)], and the second term is due to short-range diffusion from a thin shell with a thickness of the order $R_{c}$ around the capture sphere [see Fig. 1(b)]. The EPQ rate is thus expected to be time dependent. The time-averaged effective value of the rate coefficient which would follow from steadystate PL studies, $k_{\mathrm{EPQ}, \text { eff }}$ (defined more precisely below), is then expected to depend on the exciton lifetime. In as far as we know, this dependence has not yet been considered in earlier analyses. The interpretation of experimental TPQ 


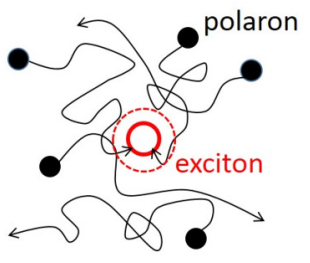

(a) 3D-diffusion

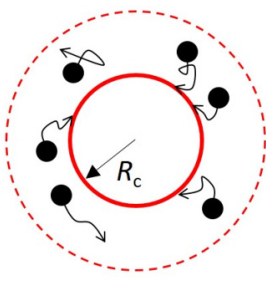

(b) quasi-1D diffusion

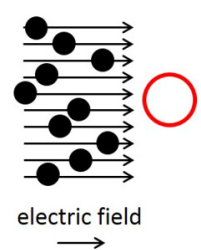

(c) drift
FIG. 1. Various contributions to exciton-polaron quenching which results from instantaneous exciton loss when a polaron arrives at a distance $R_{c}$ (capture radius) from the exciton: (a) the contribution due to long-range $3 \mathrm{D}$ diffusion, given by the first term in Eq. (2), (b) the contribution given by the second term in Eq. (2), due to quasi-1D short-range diffusion from a distance to the capture sphere of the order $\sim R_{c}$ or less (dashed circle), and (c) the "polaron wind" contribution at high electric fields, when a significant polaron (drift) current has developed.

studies may also be complicated by the filamentary nature of the current density in disordered organic semiconductor materials. The charge transport is known to be inhomogeneous at a scale of nanometers or even tens of nanometers, depending on the degree and type of disorder [18-20]. The diffusion at small time and length scales is therefore not well described by the long-distance steady-state diffusion coefficient, so that the effect of diffusion on the EPQ rate coefficient is highly nontrivial. Furthermore, one may expect that in the presence of an electric field also a charge carrier drift ("polaron wind") term contributes to the EPQ rate, as shown schematically in Fig. 1(c).

In this paper, we develop a molecular-scale approach to EPQ in disordered organic semiconductors. We focus on steady-state conditions, and show using kinetic Monte Carlo (kMC) simulations that the effective steady-state rate coefficient $k_{\mathrm{EPQ} \text {,eff }}$ depends indeed on the emissive lifetime, as already anticipated above. We consider nearest-neighbor polaron hopping in a spatially uncorrelated Gaussian density of states (DOS), and study the dependence of $k_{\mathrm{EPQ} \text {,eff }}$ on the disorder energy and on the electric field for the case of instantaneous exciton quenching when a polaron diffuses to a nearest neighbor (NN) of a molecule at which an exciton resides, as in a Dexter-type short-range quantummechanical tunneling process. We only consider EPQ due to polaron diffusion, and neglect the contribution due to exciton diffusion. Such a situation arises to a good approximation in phosphorescent OLEDs, in which the emission occurs due to triplet exciton decay on a (usually) small concentration of dye molecules. By making use of matrix molecules at which the lowest triplet energies are much larger, triplet exciton diffusion is then strongly suppressed so that the triplet-polaron encounter rate is not anymore determined by the triplet diffusion coefficient. The efficiency rolloff of prototypical green and red phosphorescent OLEDs, as obtained from kMC simulations using this immediate NN-EPQ scenario, was found to agree well with the experimental rolloff [21]. We note, however, that the actual mechanism of the triplet-polaron-quenching (TPQ) process in phosphorescent OLEDs is a subject of current debate. Aside from Dexter-type NN quenching, also TPQ due to a more long-range Förster-type dipole-dipole interaction has been regarded as relevant to organic semiconductors [22,23]. In a forthcoming paper, we will apply the approach developed in this work to analyze EPQ for such a more complex situation, where the range of the interaction (the Förster radius) constitutes an additional parameter. Furthermore, we plan to refine these studies by including longer-range polaron hopping and by studying two- and three-component host-guest systems, with fluorescent or phosphorescent emissive molecules in a single host material and in a mixed (electron transporting/hole transporting) host, respectively.

The paper is organized as follows. Section II contains a description of the simulation and analysis methods used. In Secs. III and IV, these methods are used to study EPQ in systems without and with energetic disorder, respectively, for a wide range of polaron densities, exciton emissive lifetimes, and electric fields. We find significant deviations from the predictions which would follow from the exciton-polaron capture radius model, including a significant electric field effect. Section V contains a summary and conclusions.

\section{SIMULATION AND ANALYSIS METHODS}

\section{A. Simulation approach}

We study the quenching of excitons in disordered organic semiconductor materials using the kinetic Monte Carlo (kMC) tool BUMBLEBEE [24] which has been explained extensively in earlier work on the rolloff [21,22,25], degradation [25], and triplet-triplet annihilation [26,27] in phosphorescent OLEDs. The systems considered are $100 \times 100 \times 100 \mathrm{~nm}^{3}$ boxes in which the molecular sites reside on a simple cubic lattice with a lattice constant $a$. Periodic boundary conditions are used in all three directions.

In each box, a fixed volume density of holes $n_{h}$ is present, corresponding to a fixed hole concentration $c_{h} \equiv n_{h} / N_{t}$. Here, $N_{t}=a^{-3}$ is the total volume density of molecular sites. The hole DOS is Gaussian, with a width (standard deviation) $\sigma$. The disorder is spatially uncorrelated. All simulations are performed at $T=298 \mathrm{~K}$, for $\sigma=0,0.05,0.10$, and $0.15 \mathrm{eV}$, corresponding to disorder parameters $\sigma /\left(k_{\mathrm{B}} T\right)$ equal to 0 or equal to approximately 2,4 , and 6 , respectively. Although the latter values are not exact, we use them throughout this paper to indicate the simulation results. The hole transport is described as a nearest-neighbor hopping process using the Miller-Abrahams formalism [28], which allows for successful modeling of charge transport in devices of small-molecule semiconductors [29]. The resulting charge-transport properties are very similar to those obtained with the often-used Marcus model [30,31] which would require the introduction of an additional parameter, viz., the reorganization energy. As only hops over the same distance $a$ to the six nearest-neighbor sites are included, the wave-function decay length does not enter as a parameter. For the single-component systems studied, and for disorder parameters in the range included, this is known to be a good approximation. The Coulomb interaction between the holes is switched off. Up to carrier concentrations around $10^{-2}$, that is also known to be an excellent approximation for transport in a Gaussian DOS [32]. The study includes variations of $c_{h}$ in the $10^{-5}-10^{-2}$ range. Hole concentrations 
of the order $10^{-3}$ are realistically found in the emissive layer in multilayer OLEDs under high luminance conditions. The simulations were in general carried out for a range of values of the electrostatic field $F$, applied uniformly over the box.

The boxes contain at any moment in time 1000 excitons (i.e., there is an exciton at $0.1 \%$ of all sites), which are added at random positions in the box at which no polaron already resides. The kMC simulations are used to determine the fate of these excitons: exciton-hole quenching, radiative or nonradiative decay. The latter decay processes are characterized by a decay time $\tau_{\text {rad }}$ and $\tau_{\mathrm{nr}}$, respectively. The effective exciton decay time is then $\tau=\left(1 / \tau_{\text {rad }}+1 / \tau_{\mathrm{nr}}\right)^{-1}$. The nonradiative decay channel has been included for the sake of generality. It has no effect on the overall conclusions of this study. The default values for the exciton radiative and nonradiative decay times are $\tau_{\mathrm{rad}, 0}=1.84 \times 10^{-6} \mathrm{~s}$ and $\tau_{\mathrm{nr}, 0}=5.52 \times 10^{-6} \mathrm{~s}$, so that $\tau_{0}=1.38 \times 10^{-6} \mathrm{~s}$. These are realistic values for the phosphorescent orange-red emitter bis(2methyldibenzo[f,h]quinoxaline)(acetylacetonate)-iridium(III) $\left[\operatorname{Ir}(\mathrm{MDQ})_{2}\right.$ (acac)] [25]. However, results for values of $\tau$ in the range $10^{-10}$ to $10^{-2} \mathrm{~s}$, while keeping the ratio $\tau_{\mathrm{nr}} / \tau_{\text {rad }}$ fixed, are also included.

EPQ is described as a process which occurs immediately when a hole has diffused to a nearest neighbor of a site at which an exciton resides. The exciton then jumps to this NN site, so that an excited hole is formed, followed by an immediate loss of the excess hole energy. The hole is not displaced, so that EPQ processes do not affect the mobility. All other excitonic interactions, such as exciton diffusion, exciton-exciton annihilation, or exciton degradation, have been switched off. Using Eq. (1), it follows that an effective (time-averaged) value of $k_{\mathrm{EPQ}}$ can be deduced from the fractions $f_{Q}$ and $f_{R}$ of quenched and radiatively decayed excitons, respectively, obtained from $\mathrm{kMC}$ simulations, using the expression

$$
k_{\mathrm{EPQ}, \mathrm{eff}}=\frac{1}{n_{h} \tau_{\mathrm{rad}}} \frac{f_{Q}}{f_{R}} .
$$

We note that, equivalently, the effective rate coefficient may be expressed as $k_{\mathrm{EPQ}, \text { eff }}=\left[f_{Q} /\left(f_{R}+f_{N}\right)\right] /\left(n_{h} \tau\right)$, with $f_{N}$ the fraction of nonradiatively decayed excitons.

Formally, it would be possible to express all simulation results in terms of a fundamental length unit, e.g., the nearestneighbor distance $a$, and a fundamental time unit, e.g., the average hopping time $t_{0}$ between two nearest-neighbor sites with equal hole energies. However, in order to help the reader to readily see the relevance of the results to realistic materials, we present the simulation results for concrete values of these time and length scales: $a=1 \mathrm{~nm}$, so that $N_{t}=10^{-27} \mathrm{~m}^{-3}$, a value typical for small-molecule materials used in OLEDs, and $t_{0}=3.0 \times 10^{-11} \mathrm{~s}$, a value typical for hole transport in the often-used material $\alpha-\mathrm{NPD}$ [21,33] [N, $\mathrm{N}^{\prime}$-Di(1-naphthyl)$\mathrm{N}, \mathrm{N}^{\prime}$-diphenyl-(1,1'-biphenyl)-4,4'-diamine].

During the simulations, the time dependence of the current density and quenching probability was carefully followed, in order to ensure that (i) the final results are not anymore influenced by the initial transient approach to equilibrium, and (ii) the statistical accuracy of the fractions of quenched and radiatively decayed excitons has reached a sufficiently high level. In all cases, at least five $100 \times 100 \times 100 \mathrm{~nm}^{3}$ simulation boxes are considered, in order to reduce the statistical uncertainty related to the finite system size and to accelerate the simulations by parallelization. The results were found to be insensitive to the number of excitons, up to at least 2000 excitons in the box.

\section{B. Analysis methods: Zero-field limit}

From macroscopic diffusion theory, the decrease of the exciton volume density due to EPQ in a spatially uniform system in which excitons have been generated at $t=0$ is [using Eqs. (1) and (2)] in the zero-electric-field limit given by [17]

$$
n_{E}(t)=n_{E}(0) \exp \left(-\frac{t}{\tau}-4 \pi D R_{c} n_{h} t-8 \sqrt{\pi D t} R_{c}^{2} n_{h}\right) \text {. }
$$

The decrease of the photoluminescence can thus show a distinct nonmonoexponential shape. Under favorable conditions, when the two EPQ-induced loss terms are approximately equal at $t=\tau$, it will be possible to deduce from an analysis of the time-dependent photoluminescence the separate values of the diffusion coefficient and the capture radius, if the hole density is known. The effective steady-state EPQ rate coefficient, defined by Eq. (3), is equal to

$$
k_{\mathrm{EPQ}, \mathrm{eff}}=\frac{\int_{0}^{\infty} k_{\mathrm{EPQ}}(t) n_{E}(t) d t}{\int_{0}^{\infty} n_{E}(t) d t},
$$

with $k_{\mathrm{EPQ}}(t)$ and $n_{E}(t)$ as given by Eqs. (2) and (4), respectively. This expression may be used to obtain the effective capture radius, defined as

$$
R_{c, \text { eff }} \equiv \frac{k_{\mathrm{EPQ}, \mathrm{eff}}}{4 \pi D} .
$$

For a sufficiently small hole volume density $n_{h}$ (so that $4 \pi R_{c}^{3} n_{h} \ll 1$, see below), the effective capture radius is approximately equal to

$$
R_{c, \text { eff }} \cong R_{c} \times\left(1+\frac{R_{c}}{\sqrt{D \tau}}\right) .
$$

This result shows that the value of $R_{c \text {,eff }}$ (and of $k_{\mathrm{EPQ}, \text { eff }}$ ) as deduced from a steady-state simulation or measurement can be strongly enhanced as compared to the value which would follow in the limit of a large exciton lifetime. We find that even in the large- $\tau$ limit $\left(\sqrt{D \tau} \gg R_{c}\right)$, the effective capture radius can be enhanced. For any arbitrary value of $n_{h}, R_{c \text {,eff }}$ is then given by

$$
R_{c, \text { eff }} \cong \frac{R_{c}}{1-\sqrt{\pi n_{h} R_{c}^{3}} \exp \left(4 n_{h} R_{c}^{3}\right) \operatorname{erfc}\left(\sqrt{4 n_{h} R_{c}^{3}}\right)},
$$

with erfc the complementary error function, so that for small hole densities

$$
R_{c, \text { eff }} \cong R_{c}\left[1+\sqrt{4 \pi R_{c}^{3} n_{h}}+O\left(n_{h}\right)\right] .
$$

The enhancement thus occurs for high hole densities, when the probability of finding a hole in a volume of the order of the capture sphere volume is no longer much smaller than 1. We indeed find this effect from the kMC simulations, as 
will be shown in Secs. III and IV. The enhancement may be viewed as the result of a crossover to a regime in which the quench rate is increased due to a "competition" between various nearby polarons. From kMC simulations [26] and from experiment [27] we have found an analogous high-density enhancement of the rate coefficient for steady-state excitonexciton annihilation occurring at high exciton densities.

We find that this continuum approach is not in all cases a good first starting point, as it does not include the finite probability $p_{\mathrm{NN}}$ that after exciton generation immediate quenching takes place due to the presence of a polaron on a nearestneighbor lattice site. This contribution is only important when the exciton lifetime is very short, so that only very-short-range diffusion processes contribute to the quenching. We model the effect by assuming that the exponential decay of the volume density of the remaining excitons is not affected by the immediate exciton loss due to NN quenching. The effective time-averaged steady-state EPQ rate coefficient, defined by Eq. (3), is then equal to

$$
k_{\mathrm{EPQ}, \mathrm{eff}}=\frac{p_{\mathrm{NN}}+\left(1-p_{\mathrm{NN}}\right) \int_{0}^{\infty} k_{\mathrm{EPQ}}(t) n_{h} \frac{n_{E}(t)}{n_{E}(0)} d t}{\left(1-p_{\mathrm{NN}}\right) \int_{0}^{\infty} n_{h} \frac{n_{E}(t)}{n_{E}(0)} d t},
$$

with $k_{\mathrm{EPQ}}(t)$ and $n_{E}(t)$ as given by Eqs. (2) and (4), respectively.

\section{Analysis methods: Electric-field dependence}

In the presence of an electric field, the theoretical description of the EPQ process is complicated by the fact that there will be a polaron drift (or "polaron wind") contribution to the EPQ rate. In the emissive layer of OLEDs, e.g., the internal field can be as large as $2 \times 10^{7} \mathrm{~V} / \mathrm{m}$ or more at the highest luminance levels used in practical display or lighting systems. It is then convenient to describe the effect of EPQ in terms of an effective EPQ capture cross section, which is defined as

$$
A_{c} \equiv \frac{r_{\mathrm{EPQ}, \mathrm{eff}}}{J / e}=\frac{k_{\mathrm{EPQ}, \mathrm{eff}} n_{h}}{J / e} .
$$

Using Eq. (3), $A_{c}$ may be obtained from the fractions of quenched and radiatively decayed excitons using

$$
A_{c}=\frac{e}{J \tau_{\text {rad }}} \frac{f_{Q}}{f_{R}} .
$$

If for high fields the drift velocity would increase limitless, so that the role of lateral diffusion would become negligible, the EPQ process could be depicted as in Fig. 1(c). For the nearestneighbor EPQ scenario considered, $A_{\mathrm{c}}$ would then be equal to $5 \mathrm{~nm}^{2}$. That is the area formed by the site at which the exciton resides plus the four nearest-neighbor sites which reside in the plane perpendicular to the electric-field direction. However, Fig. 1(c) is actually oversimplified, as at high fields the forward hops are almost all downhill (to a lower-energy final state) and therefore no longer thermally activated. Within the MillerAbrahams formalism assumed, the drift velocity then saturates, so that diffusion will even at high fields continue to play a role: a polaron which otherwise would miss the capture region can with a finite probability due to lateral diffusion still give rise to a hit. The effective EPQ capture cross section in the infinite field limit will therefore be larger than $5 \mathrm{~nm}^{2}$.
For a system without disorder, the drift velocity $v(F)$ is proportional to $\left[1-\exp \left(-e a F / k_{\mathrm{B}} T\right)\right]$. In the limit of a very small capture radius, the capture cross section is expected to be inversely proportional to $v(F)$, and to be given by

$$
A_{c}(F) \cong \frac{A_{c, \infty}}{1-\exp \left(-\frac{F}{F_{0}}\right)},
$$

with $A_{c, \infty}$ the value of $A_{c}$ in the high-field limit and with $F_{0}=$ $k_{\mathrm{B}} T /(e a)$, i.e., $2.57 \times 10^{7} \mathrm{~V} / \mathrm{m}$ at $298 \mathrm{~K}$. Before investigating the applicability of this expression to the case of NN-type EPQ (in Secs. III and IV), we have first studied the more simple case of immediate quenching when a polaron hops (with a rate unaffected by the presence of the exciton) to the excited molecule itself. We find that Eq. (13) indeed provides a good fit to kMC simulation data for this case. For this case, the drift-only value for $A_{c, \infty}$ would be $1 \mathrm{~nm}^{2}$, the geometrical area of one lattice site. However, the actual value as obtained from the $\mathrm{kMC}$ simulations (which include the effect of polaron diffusion) is much larger, viz., $3.94 \pm 0.01 \mathrm{~nm}^{2}$.

The capture cross section is in the following way related to the capture radius, discussed in the previous subsection. For very small fields, it follows from Eqs. (6) and (11) that

$$
A_{c}(F)=\frac{4 \pi D R_{c} n_{h}}{J / e}=4 \pi \frac{k_{\mathrm{B}} T}{e} R_{c, \text { eff }} \frac{1}{F} .
$$

The second step has been made using that $J=n_{h} e \mu\left(n_{h}\right) F$, and using the classical Einstein equation

$$
D=\frac{k_{\mathrm{B}} T}{e} \mu_{h},
$$

with $k_{\mathrm{B}}$ the Boltzmann constant, $T$ the temperature, and $e$ the fundamental charge. However, we will show that for intermediate fields (when $A_{c}$ is not anymore much larger than the geometrical cross section of $5 \mathrm{~nm}^{2}$ mentioned above) a correction is needed, related to the polaron wind effect, and that $A_{c}$ may then be described more accurately by

$$
A_{c}(F) \cong 4 \pi \frac{k_{\mathrm{B}} T}{e} R_{c, \text { eff }}^{\prime} \frac{1}{F}+A_{c, \infty}^{\prime} .
$$

The first and second terms may be viewed as a diffusion and polaron wind contribution, respectively. The effective capture radius $R_{c \text {,eff }}^{\prime}$ is primed, in order to indicate that it refers to an analysis using Eq. (16) of the capture cross section obtained for a range of values of the field. $R_{c \text {,eff }}^{\prime}$ is, e.g., different from $R_{c \text {,eff }}$ if (for very small $D$ ) immediate quenching predominantly determines $R_{c \text {,eff }}$ (see the previous subsection). Another complication which could give rise to a difference is the field dependence of the diffusion coefficient, which moreover is in general a tensor with for the case of systems with Gaussian disorder a longitudinal value which is larger than the transverse value [34,35]. For systems without disorder, for which (as discussed above) the mobility is already field dependent at small fields, Eq. (13) provides therefore a better description than Eq. (16). The second term, $A_{c, \infty}^{\prime}$, is the effective cross section as extrapolated linearly to the high-field limit. Also, this parameter is primed, in order to distinguish it from the actual value in the high-field limit. For energetically disordered systems, $A_{c, \infty}^{\prime}$ is found from the $\mathrm{kMC}$ simulations to be close to the geometrical value of $5 \mathrm{~nm}^{2}$, as will be shown in Sec. IV. 


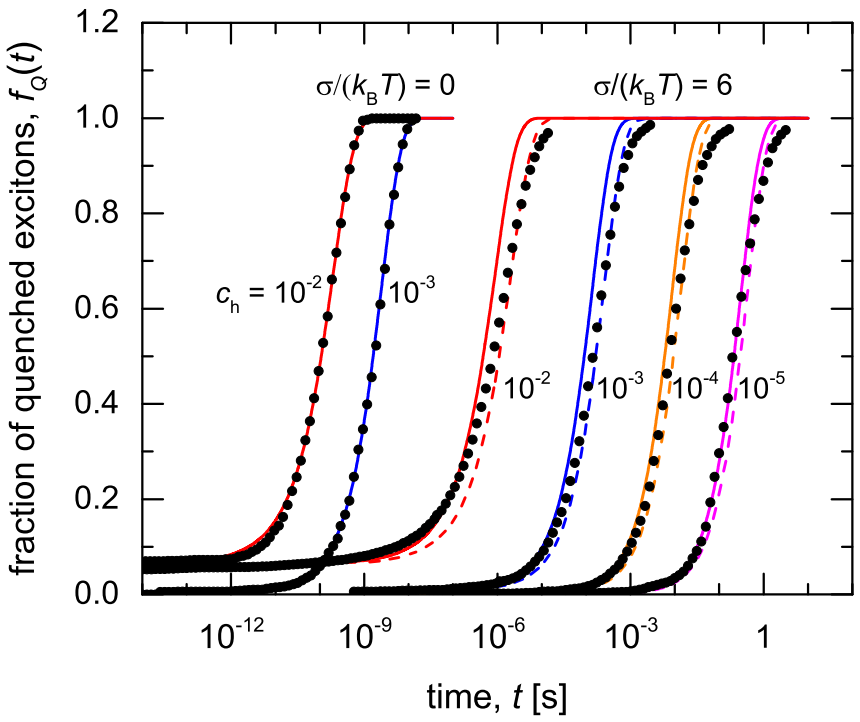

FIG. 2. Time dependence of the total fraction of quenched excitons, placed at random positions in materials with various hole polaron concentrations $c_{h}$ and with disorder strengths $\sigma /\left(k_{\mathrm{B}} T\right)=0$ (no energetic disorder) and $\sigma /\left(k_{\mathrm{B}} T\right)=6$, as obtained from $\mathrm{kMC}$ simulations. The exciton lifetime is taken to be infinite, and the field $F=0$. The curves give the fractions of quenched excitons expected from Eq. (10), with the diffusion coefficient as obtained from the low-field mobility using the generalized Einstein equation (full curves) and the classical Einstein equation (dashed curves).

\section{SIMULATION RESULTS: SYSTEMS WITHOUT ENERGETIC DISORDER}

In this section, we study EPQ in systems without energetic disorder, i.e., $\sigma /\left(k_{\mathrm{B}} T\right)=0$. As a first step, we study the EPQ process in a time-resolved manner, by calculating the time-dependent total fraction of quenched excitons $f_{Q}(t)$ for the case of an infinite exciton lifetime. This fraction follows directly from the fraction of emitted excitons, given by the exponential factor in Eq. (4) with $1 / \tau=0$, corrected by the probability $p_{\mathrm{NN}}$ that after exciton generation immediate quenching takes place due to a polaron on a nearest-neighbor site:

$f_{Q}(t)=1-\left(1-p_{\mathrm{NN}}\right) \exp \left(-4 \pi D R_{c} n_{h} t-8 \sqrt{\pi D t} R_{c}^{2} n_{h}\right)$.

In Fig. 2, $f_{Q}(t)$ is shown for several cases, as obtained using Eq. (17) (full curves) and as obtained from $\mathrm{kMC}$ simulations at very small electric fields (symbols). We focus here on the left part of the figure, which gives the results for systems without disorder and hole concentrations equal to $10^{-2}$ and $10^{-3}$. Good agreement between the theoretical and simulation data is obtained with $R_{c}=0.92 \pm 0.02 \mathrm{~nm}$, $p_{\mathrm{NN}} \cong 6 n_{h} / N_{t}$, and using the value of the diffusion coefficient $D=(3.25 \pm 0.10) \times 10^{-8} \mathrm{~m}^{2} / \mathrm{s}$ that follows from the hole mobility $\mu_{h}$ using the classical Einstein equation [Eq. (15)]. The mobility has been deduced from the current density $J$ as obtained at a low electric field $F$ using the expression $\mu_{h}=J /\left(n_{h} e F\right)$. The value of $D$ obtained in this manner is consistent with the theoretical value that follows from the hopping attempt frequency $t_{0}^{-1}$ and the intersite distance $a$,

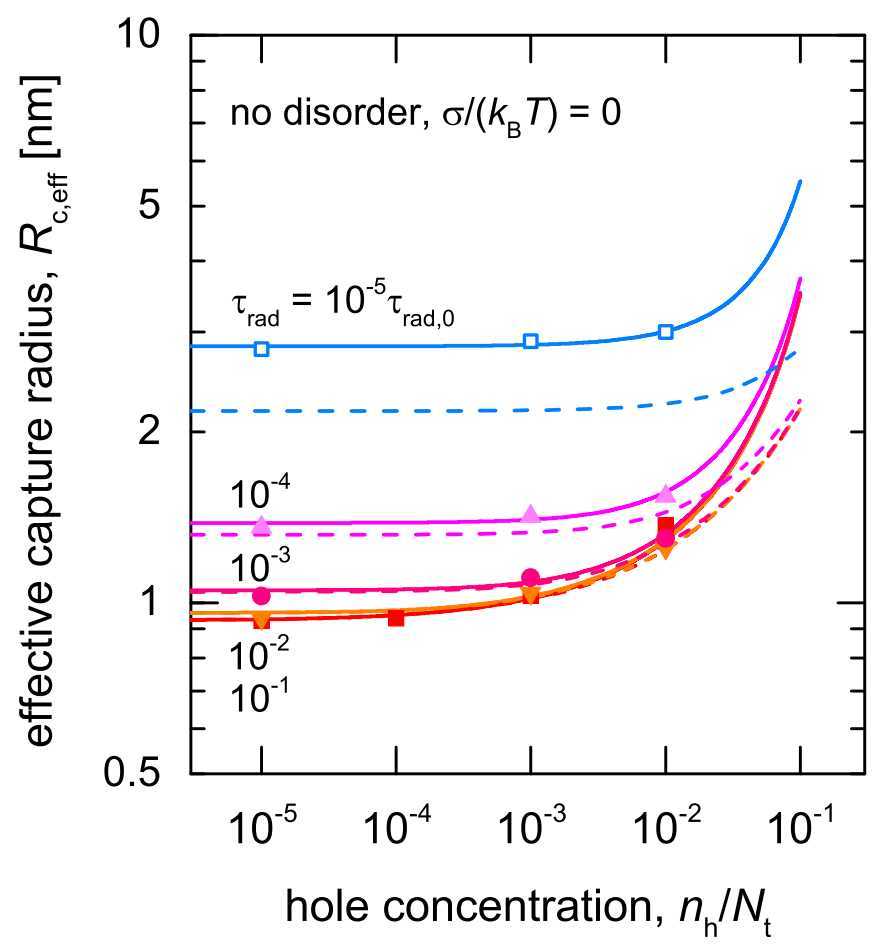

FIG. 3. Dependence of the effective steady-state EPQ capture radius for the case of immediate $\mathrm{NN}$-type $\mathrm{EPQ}$ on the hole concentration and the radiative lifetime, for systems without energetic disorder. The symbols give the $\mathrm{kMC}$ simulation results (see Table I for the numerical values). The full curves give a theoretical fit using Eqs. (6) and (10), with a capture radius $R_{c}=0.92 \mathrm{~nm}$ and a probability $p_{\mathrm{NN}} \cong 3.7 n_{h} / N_{t}$ of immediate quenching at a $\mathrm{NN}$ polaron. The dashed curves give, for the same capture radius, the theoretical curves obtained with $p_{\mathrm{NN}}=0$, i.e., neglecting immediate NN quenching.

$D=a^{2} / t_{0}=3.33 \times 10^{-8} \mathrm{~m}^{2} / \mathrm{s}$. The value of $R_{c} \cong 0.92 \mathrm{~nm}$ that is obtained from the fit is close to the value of about $1 \mathrm{~nm}$ expected from the assumption of nearest-neighbor quenching. The results obtained for disordered systems, which show a distinct deviation, will be discussed in Sec. IV.

Figure 3 shows the effective capture radius as obtained from $\mathrm{kMC}$ simulations (symbols), for hole concentrations in the range $10^{-5}$ to $10^{-2}$ and for exciton radiative lifetimes ranging from $10^{-1}$ to $10^{-7}$ times the default value of $\tau_{0}=1.38 \times 10^{-6} \mathrm{~s}$ (see Sec. II A). The simulations were carried out at a small but finite field $\left(F=5 \times 10^{5} \mathrm{~V} / \mathrm{m}\right)$. The mobility and diffusion coefficient were found to be independent of the carrier density and exciton lifetime, as expected, and were within the statistical accuracy equal to the theoretically expected values. The values of the capture radius shown in the figure were obtained after extrapolation to zero electric field, in a manner described in the Appendix. This extrapolation constitutes only a very small correction. Table II in the Appendix gives an overview of the simulation data and of the analysis results. The full curves give the effective capture radius [Eq. (6)], as obtained using Eq. (10) with the value of $R_{c}=0.92 \pm 0.02 \mathrm{~nm}$ deduced above and with $p_{\mathrm{NN}}=3.7 n_{h} / N_{t}$. The latter value is smaller than the value of $p_{\mathrm{NN}}=6 n_{h} / N_{t}$ expected for small hole densities $\left(n_{h} \ll 1\right)$ for the simple cubic lattice considered. In view of 


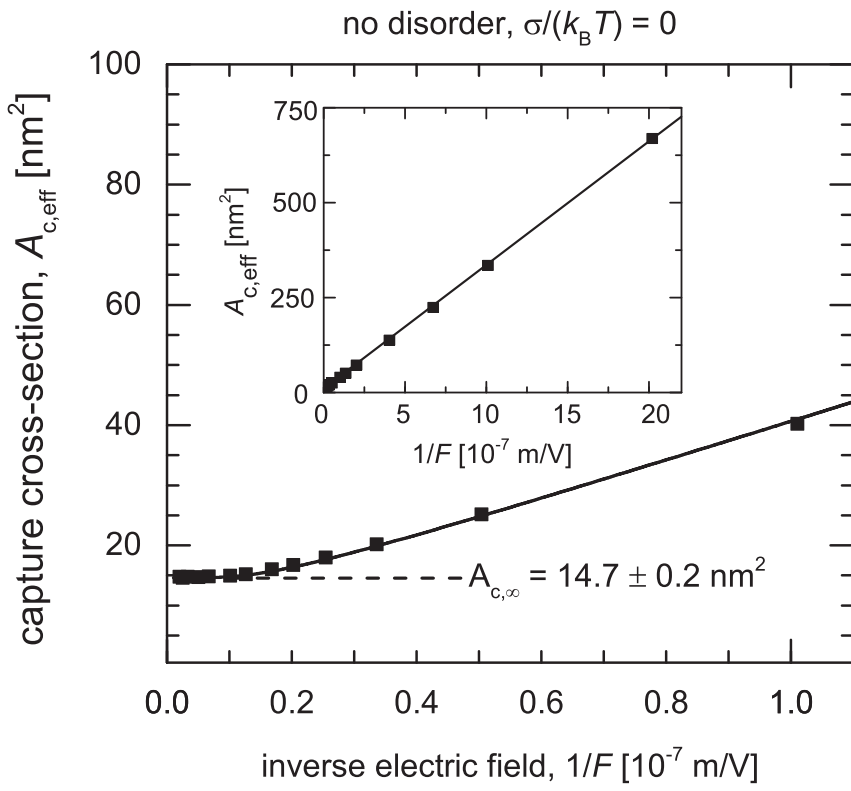

FIG. 4. Effective EPQ capture cross section as a function of the inverse of the electric field $1 / F$ for systems without energetic disorder, as obtained from $\mathrm{kMC}$ simulations (symbols) for the large- $\tau$ and small- $n_{h}$ limits. The full curve gives a best fit to the data using Eq. (13). The inset shows the simulation data and the fit result for small fields.

the approximations made, we regard the agreement as fair. The dashed curves give the effective capture radius as obtained with $p_{\mathrm{NN}}=0$. It is evident that including the immediate quenching effect in a manner as described above provides a considerable improvement of the fit to the data for small exciton lifetimes.

In the large- $\tau$ limit, the figure reveals for $n_{h} \cong 10^{-3}$ and larger an increase of the effective capture radius with increasing polaron density. Also, this high-density effect, which was already discussed and explained in Sec. II B [Eq. (9)], is consistently described using the theoretical framework developed in Sec. II B.

Figure 4 shows the EPQ capture cross section as obtained from $\mathrm{kMC}$ simulations (symbols) as a function of the inverse electric field $1 / F$ for the large- $\tau$ and small- $n_{h}$ limits. In the high-field limit, $A_{c}$ is found to saturate in a manner as described well by Eq. (13) (full curve). The high-field value of the capture cross section is $14.7 \pm 0.2 \mathrm{~nm}^{2}$, which is almost a factor of 3 larger than the value of $5 \mathrm{~nm}^{2}$ expected from a drift-only model. The best-fit value of $F_{0}$ is $2.25 \times 10^{7} \mathrm{~V} / \mathrm{m}$, i.e., slightly smaller than the value expected for the small-capture radius limit. For very small electric fields, $A_{c}$ is to a good approximation proportional to $(1 / F)$, as may be seen from the inset in the figure.

\section{SIMULATION RESULTS: EFFECTS OF DISORDER}

In this section, we use kMC simulations to investigate the effect of energetic disorder on the EPQ rate, for systems with disorder strengths up to $\sigma /\left(k_{\mathrm{B}} T\right)=6$. We study the EPQ rate as a function of the emissive lifetime, polaron density, and electric field. We have most extensively studied systems with a hole concentration of $10^{-3}$ and with $\sigma /\left(k_{\mathrm{B}} T\right)=4$, a value
TABLE I. Hole self-diffusion coefficient in organic semiconductors with Gaussian energetic disorder, as obtained from kMC simulations at $298 \mathrm{~K}$ from the current density under low-field conditions using the classical Einstein equation [Eq. (15)] for various values of the disorder strength $\sigma /\left(k_{\mathrm{B}} T\right)$ and hole concentration $n_{h}$. The simulations were carried out at $F=10^{7} \mathrm{~V} / \mathrm{m}$ for the disordered systems, and at $F=10^{6} \mathrm{~V} / \mathrm{m}$ for $\sigma /\left(k_{\mathrm{B}} T\right)=0$. The numerical uncertainty is approximately $3 \%$ for systems with $\sigma /\left(k_{\mathrm{B}} T\right)=0$ and 2 and increases with increasing disorder strength and decreasing concentration to about $6 \%$ for $\sigma /\left(k_{\mathrm{B}} T\right)=6$ and $c_{h}=10^{-5}$. Unit: $10^{-10} \mathrm{~m}^{2} / \mathrm{s}$.

\begin{tabular}{lcccc}
\hline \hline$\sigma /\left(k_{\mathrm{B}} T\right)$ & $c_{h}=10^{-5}$ & $c_{h}=10^{-4}$ & $c_{h}=10^{-3}$ & $c_{h}=10^{-2}$ \\
\hline 0 & 325 & 325 & 325 & 325 \\
2 & 32.5 & 32.7 & 33.3 & 36.9 \\
4 & 0.180 & 0.240 & 0.430 & 1.20 \\
6 & 0.00020 & 0.00062 & 0.0031 & 0.032 \\
\hline
\end{tabular}

which is typical for the density of highest occupied molecular orbital (HOMO) states in amorphous hole conducting semiconductors used in OLEDs. Table I gives an overview of the diffusion coefficients of the systems studied. Their values vary over more than six orders of magnitude. We investigate whether at small fields the effective capture radius, defined by Eq. (6), can be described well using Eq. (10), and whether the field dependence of the effective capture cross section is well described using Eq. (16).

\section{A. Time-dependent fraction of quenched excitons}

The validity of the model developed in Sec. II B, leading to the effective rate coefficient given by Eq. (10), may be judged by studying the time dependence of the fraction of quenched excitons $f_{Q}(t)$. Within the framework of the model, $f_{Q}(t)$ is given by Eq. (17). For systems without disorder, the model was found to provide a good description of $\mathrm{kMC}$ simulation results (left part of Fig. 2). In the right part of Fig. 2, the simulation results are given for systems with strong energetic disorder $\left[\sigma /\left(k_{\mathrm{B}} T\right)=6\right]$ and for hole concentrations ranging from $10^{-5}$ to $10^{-2}$. A comparison is given with the theoretical curves as given by Eq. (17), with the diffusion coefficient as derived from the simulated mobility using the generalized Einstein equation (full curves) or the classical Einstein equation (dashed curves).

The generalized Einstein equation provides the diffusion coefficient which describes the Fickian transport under the influence of a concentration gradient. In energetically disordered materials, this diffusion coefficient is enhanced at large carrier concentrations [36] as compared to the value given by the classical Einstein equation, which describes self-diffusion under thermal equilibrium conditions. It is not a priori clear which diffusion coefficient should be used. The figure shows that for both choices the model provides a fair approximate description of the $f_{Q}(t)$ curves, in particular concerning the time at which half of the excitons have been quenched. However, the simulated curves are somewhat less steep, and show significant deviations at very short and very long times. The difference between the two models is largest for a hole concentration of $10^{-2}$, for which the two diffusion coefficients differ by a factor 2.31 . 
The figure suggests that at short times the quenched fraction is more accurately predicted using the generalized Einstein equation. That might be rationalized by considering that in the small region formed by the six NN sites around the target site there are no polarons. There is thus, effectively, a local polaron density gradient. A different way of understanding the simulation result is by considering that (on average) long-range steady-state diffusion is more strongly hindered by the disorder than the more local diffusion on a short time scale. As a result, the ac mobility (and diffusivity) of disordered systems shows at high frequencies an increase with increasing frequency [37], and the effective (ensemble-averaged) dc conductivity increases when the system size is reduced to a value below a certain critical disorder-dependent length scale [19].

For long diffusion times, one might expect that the relevant diffusion process is just self-diffusion, in the absence of a concentration gradient, so that the classical Einstein equation is more relevant. Indeed, for times at which around $90 \%$ of all excitons have been quenched, this provides a good prediction. However, for even larger times the fraction of quenched excitons is smaller than as expected from the model. That might be viewed as a result of the occurrence of rare environments in which the NN sites of the exciton have a large ionization potential, forming a "protective shell" around the exciton. These deviations from the model developed in Sec. II are expected to give rise to distinct deviations of the effective capture radius, deduced from the fraction of excitons lost under steady-state conditions. Before demonstrating this effect from explicit kMC simulations (see Fig. 8), we discuss first how the results of such simulations at finite electric fields may be analyzed, so that a proper extrapolation to the zero-field limit may be made.

\section{B. Electric-field dependence of the EPQ rate}

Figures 5(a)-5(d) show the calculated dependence of the EPQ rate coefficient on the electric field for systems with various disorder strengths $\sigma /\left(k_{\mathrm{B}} T\right)$ for a hole density $n_{h}=$ $10^{24} \mathrm{~m}^{-3}$ (concentration $10^{-3}$ ) and for wide ranges of the radiative decay time $\tau_{\text {rad }}$. For all curves with $\sigma /\left(k_{\mathrm{B}} T\right)=4$ and for all cases with $\tau_{\text {rad }}=\tau_{\text {rad }, 0}$ the simulations were carried out for fields corresponding to a voltage drop ranging from 1 to $40 \mathrm{~V}$ per $100 \mathrm{~nm}$. Although due to strong Joule heating fields above $10^{8} \mathrm{~V} / \mathrm{m}$ can in practice often not easily be reached in OLEDs, we have included some high-field simulation studies, as the results might be of interest under the transient conditions which may be used in electrically induced organic lasing. For the other cases, the field range considered corresponds to a voltage drop of 1 to $5 \mathrm{~V}$ per $100 \mathrm{~nm}$.

The simulation results show that $k_{\mathrm{EPQ}}$ decreases with increasing disorder strength, as expected from the decrease of the mobility and diffusion coefficient with increasing disorder strength [38]. Furthermore, $k_{\mathrm{EPQ}}$ increases with increasing electric field. As already discussed in Sec. II, the observed saturation of the increase above a certain critical field can be understood from the well-known saturation of the drift velocity. For systems with Gaussian energetic disorder, the saturation occurs when the electric potential drop per intersite distance, eaF, becomes larger than approximately $4 \sigma$ [38].

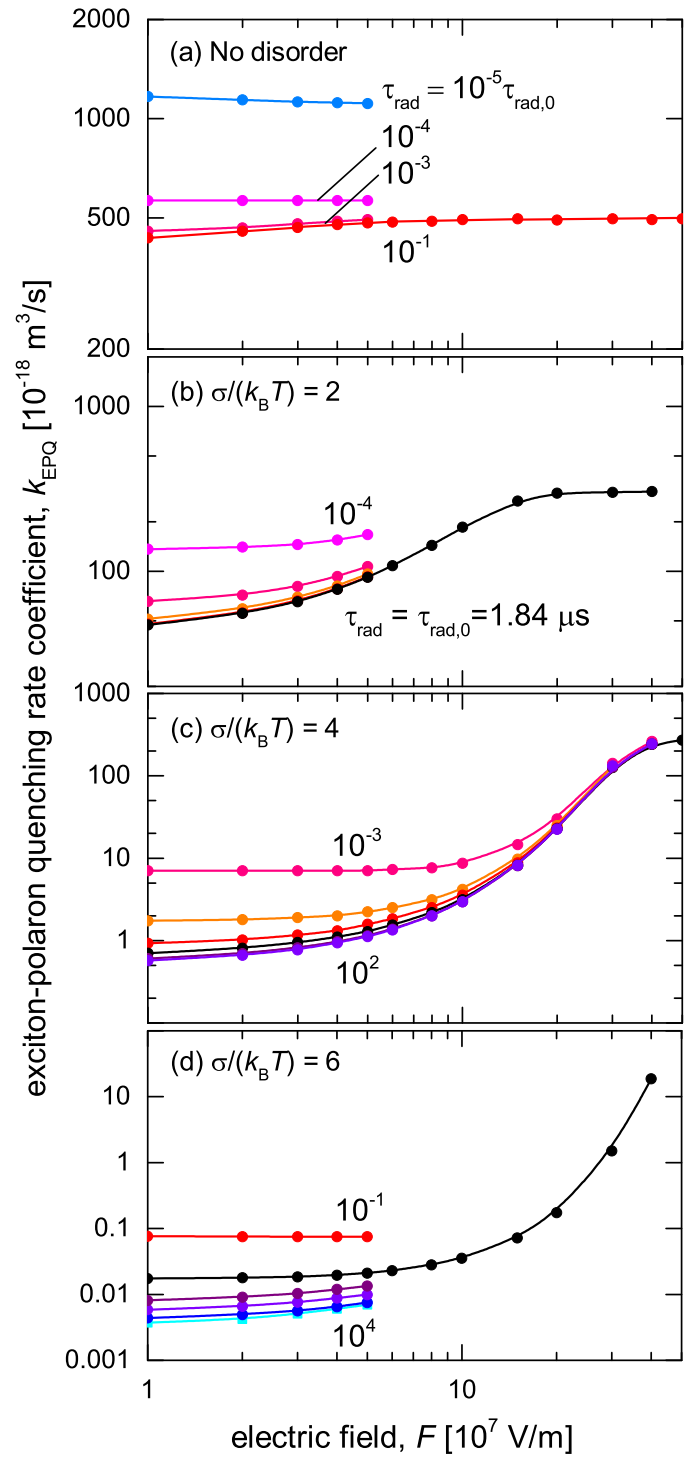

FIG. 5. (a)-(d) Electric field $(F)$ and radiative decay time $\left(\tau_{\text {rad }}\right)$ dependence of the exciton-polaron-quenching rate coefficient $k_{\mathrm{EPQ}}$ in systems with a variable uncorrelated Gaussian energetic disorder strength $\sigma /\left(k_{\mathrm{B}} T\right)$ for the case of immediate nearest-neighbor quenching, as obtained from kinetic Monte Carlo simulations. The simulations were carried out for a polaron density equal to $10^{24} \mathrm{~m}^{-3}$, and for a polaron hopping attempt time equal to $3 \times 10^{-11} \mathrm{~s}$ (see the main text). In (a), the radiative decay time $\tau_{\text {rad }}$ is equal to $\left(10^{-5}, 10^{-4}\right.$, $10^{-3}$, and $\left.10^{-1}\right) \times \tau_{\text {rad }, 0}$. In (b)-(d), $\tau_{\text {rad }}$ varies in steps of a factor 10 with respect to the default value $\tau_{\text {rad, } 0}=1.84 \mu \mathrm{s}$, as indicated.

For $\sigma /\left(k_{\mathrm{B}} T\right)=2$ and 4 , the expected critical fields, $2 \times 10^{8}$ and $4 \times 10^{8} \mathrm{~V} / \mathrm{m}$, fall in the range included in our study, and are consistent with the observed values.

Figure 5 also shows that, for all systems studied, $k_{\mathrm{EPQ}}$ depends below a certain crossover value strongly on the radiative lifetime. For $\sigma /\left(k_{\mathrm{B}} T\right)=4$, e.g., this happens for $\tau_{\text {rad }}$ smaller than approximately $10^{-5} \mu$ s, i.e., approximately $10 \times \tau_{\text {rad, } 0}$. The observed increase of this crossover lifetime with increasing disorder strength is due to the decrease of the diffusion coefficient with increasing disorder strength, so that the range over which relevant polaron diffusion occurs 


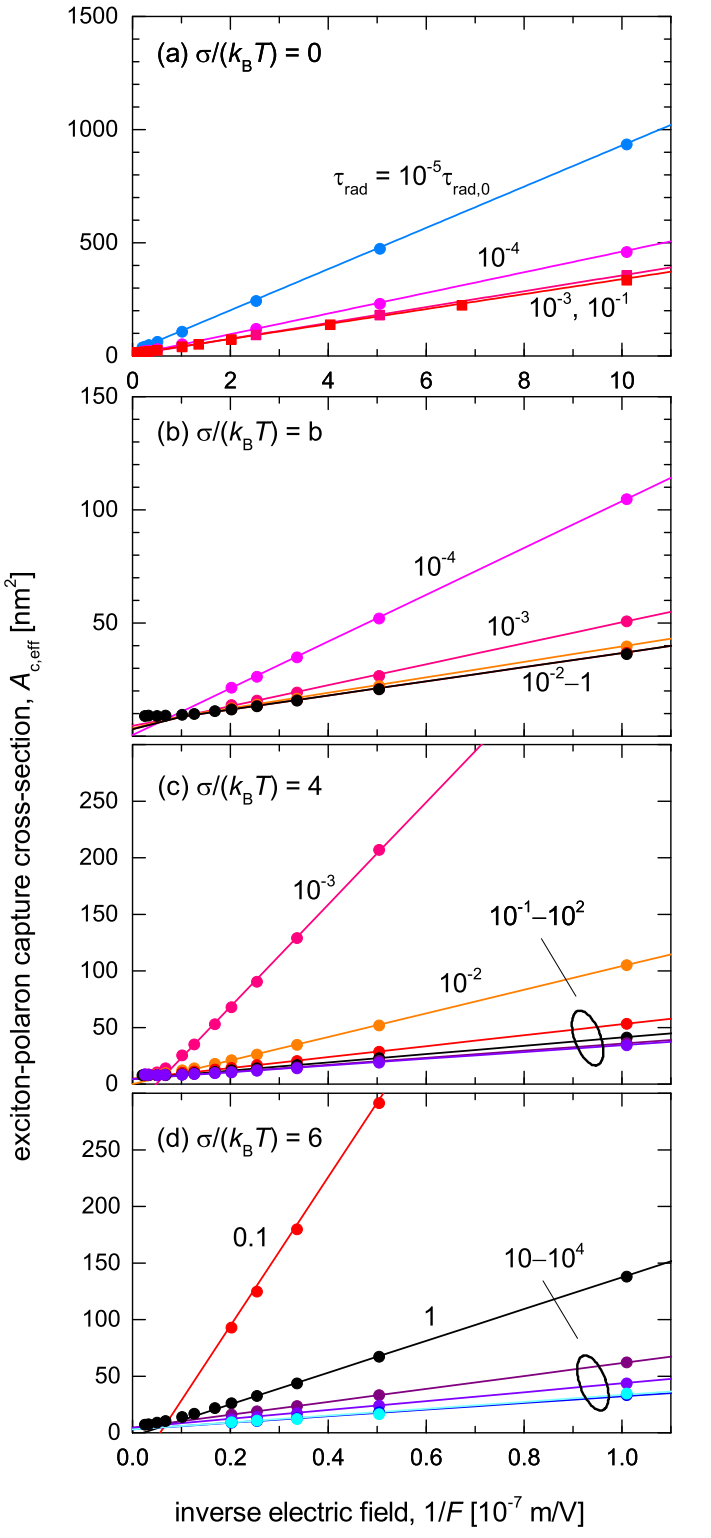

FIG. 6. (a)-(d) Exciton-polaron-quenching capture cross section as a function of the inverse electric field $1 / F$ for the case of nearest-neighbor quenching in systems with an uncorrelated Gaussian energetic disorder strength $\sigma / k_{\mathrm{B}} T=0,2,4$, and 6 , respectively, for a hole density equal to $10^{24} \mathrm{~m}^{-3}\left(c_{h}=10^{-3}\right)$. In each panel, results are given for various exciton radiative decay times, with values (given relative to $\tau_{\text {rad }, 0}=1.84 \mu \mathrm{s}$ ) which are varied in steps of a factor 10 . See the caption of Fig. 5 for simulation details.

decreases and the relative role of pseudo-1D short-range diffusion is enhanced [see Eq. (7)]. One therefore also expects a smaller electric-field dependence, which is indeed revealed by the simulation results.

A more quantitative analysis of the electric-field dependence of the EPQ rate coefficient may be given using the capture cross-section formalism, introduced in Sec. II C. From Eq. (16), the capture cross section $A_{c}$ is for small and intermediate fields expected to vary linearly with $1 / F$. Figure 6 shows the $1 / F$ dependence of the capture cross section, obtained from the rate coefficients shown in Fig. 5 and

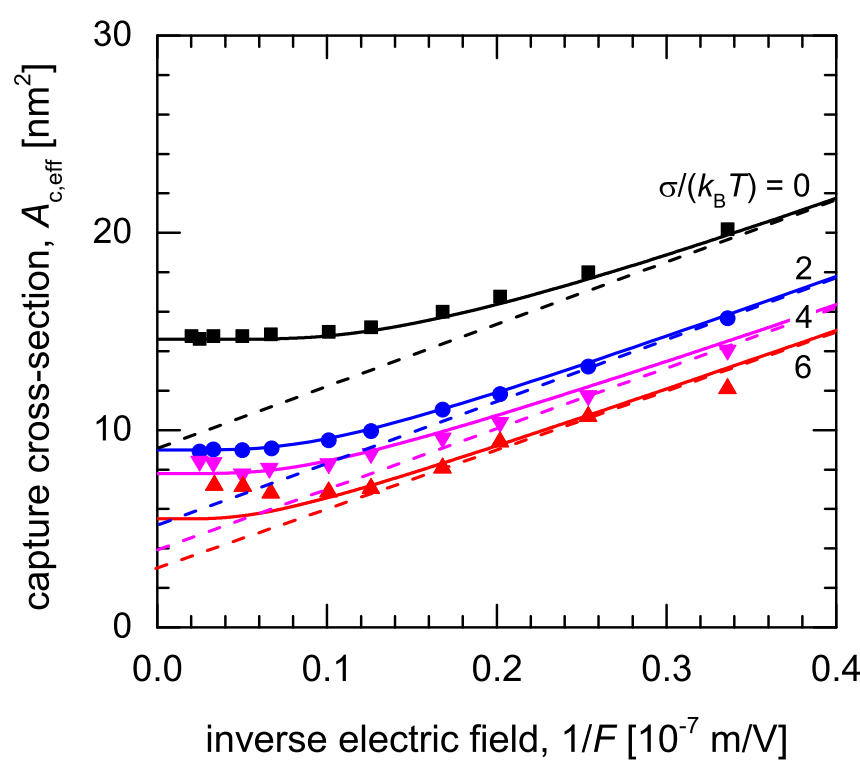

FIG. 7. Exciton-polaron-quenching capture cross section as a function of the inverse field $1 / F$ for various values of the disorder strength $\sigma /\left(k_{\mathrm{B}} T\right)$, for a hole density equal to $10^{24} \mathrm{~m}^{-3}\left(c_{h}=10^{-3}\right)$ and for the largest exciton radiative lifetimes $\tau_{\text {rad }}$ considered in our study (see Fig. 5). The figure focuses on the kMC results for large electric fields. The full curves show an empirical fit [Eq. (13)] to the high-field part of the data, and the dashed lines give the linear fit [Eq. (16)] to the low-field data shown already in Fig. 6.

from the calculated current density, for systems with disorder parameters $\sigma /\left(k_{\mathrm{B}} T\right)$ in the range 0 to 6 . For $\sigma /\left(k_{\mathrm{B}} T\right)=0$, simulations at reduced fields were included, enabling an extension of the analysis to a tenfold enlarged range of inverse field values. We note that both scales in Fig. 6(a) are extended by a factor of 10 as compared to those in Figs. 6(b)-6(d), so that the slopes of the fit lines through the data points are directly comparable. The figure shows that for sufficiently small fields, the capture radii vary indeed to an excellent approximation linearly with $1 / F$, as suggested in Sec. II C.

Figure 7 shows the capture cross section for systems with a hole concentration of $10^{-3}$, for the largest exciton lifetimes considered in our study. The results are then almost independent of $\tau_{\text {rad }}$. The figure focuses on the data obtained at small $1 / F$ (high fields). For $\sigma /\left(k_{\mathrm{B}} T\right)$ up to $4, A_{c}$ becomes field independent for the values of the field included in the study, but for $\sigma /\left(k_{\mathrm{B}} T\right)=6$ an increase of the cross section for very high fields is found. We regard this result as an indication that due to the field-induced detrapping, the lateral diffusion becomes more similar to that in a material without disorder. For $\sigma /\left(k_{\mathrm{B}} T\right)=2$ to 6 , the linear parts of the capture cross-section curves extrapolate to values $A_{c, 0}^{\prime}$ which are quite close to the geometrical value of $5 \mathrm{~nm}^{2}$. We regard the small deviations from this value as within the uncertainties of the simulations.

\section{Effective EPQ capture radius}

From the analysis given in the previous subsection, it follows that the electric-field dependence of the capture cross section is quite accurately given by Eq. (16), with $A_{c, \infty}^{\prime}=$ $5 \mathrm{~nm}^{2}$. We have used this result to obtain the dependence of 
(a) $\sigma /\left(k_{\mathrm{B}} T\right)=2$

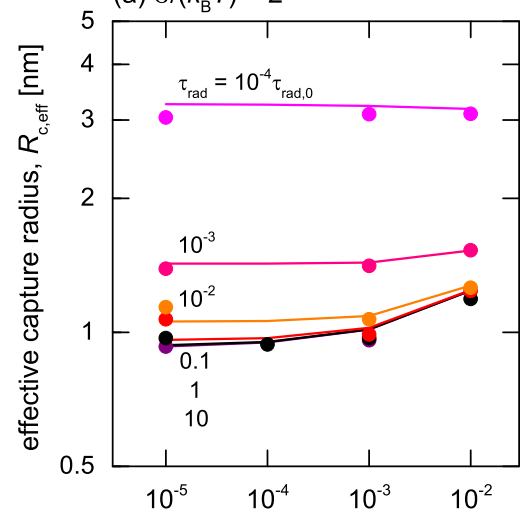

(b) $\sigma /\left(k_{\mathrm{B}} T\right)=4$

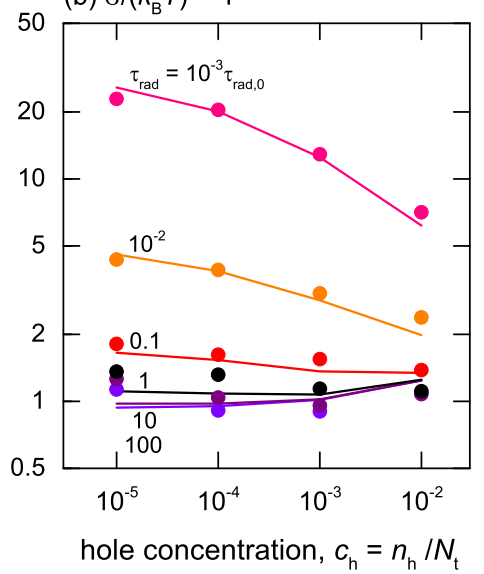

(c) $\sigma /\left(k_{\mathrm{B}} T\right)=6$

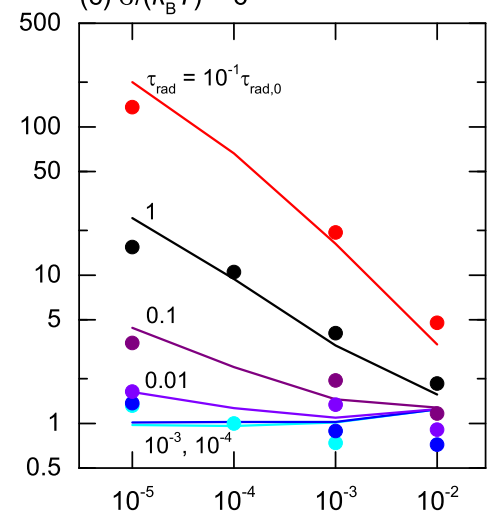

FIG. 8. Dependence of the effective steady-state EPQ capture radius for the case of immediate NN-type EPQ on the hole concentration and the radiative lifetime, for systems with $\sigma /\left(k_{\mathrm{B}} T\right)=2(\mathrm{a}), 4(\mathrm{~b})$, and 6 (c). The symbols give the kMC simulation results (see Tables III-V in the Appendix for the numerical values). The full lines connect points which are obtained from a theoretical fit using Eqs. (6) and (10), with the diffusion coefficient as given in Table I, a capture radius $R_{c}=0.92 \mathrm{~nm}$ and a NN immediate quenching probability $p_{\mathrm{NN}}=6 n_{h} / N_{t}$.

the effective capture radius in the zero-field limit on the hole concentration, the radiative lifetime, and the disorder energy from the value of the capture cross section at $F=10^{7} \mathrm{~V} / \mathrm{m}$, using the expression

$$
R_{c, \text { eff }}=\frac{k_{\mathrm{EPQ}, \mathrm{eff}}}{4 \pi D}-\frac{A_{c, \infty}^{\prime} e F}{4 \pi k_{\mathrm{B}} T} .
$$

The results (symbols) are shown in Figs. 8(a)-8(c), for $\sigma /\left(k_{\mathrm{B}} T\right)=2,4$ and 6 . The full lines connect points which are obtained from a theoretical fit using Eqs. (6) and (10), with a capture radius $R_{c}=0.92 \mathrm{~nm}$ (as used for systems without disorder in Fig. 3) and a NN immediate quenching probability $p_{\mathrm{NN}} \cong 6 n_{h} / N_{t}$. Tables II-V in the Appendix give an overview of the simulation data and the analysis results. The Appendix also provides provides further simulation and analysis details.

The figure reveals three distinct effects of energetic disorder on the effective capture radius. First, the enhancement of the capture radius with decreasing emissive lifetime becomes stronger with increasing disorder, as due to the decreasing diffusion coefficient the effect of quasi-1D diffusion [Fig. 1(b), the second term in Eq. (2)] becomes more important. The enhancement is already about one order of magnitude for typical fluorescent systems $\left(\tau_{\mathrm{rad}} \sim 1 \mathrm{~ns}\right)$ with $\sigma /\left(k_{\mathrm{B}} T\right)=4$, and for typical phosphorescent systems $\left(\tau_{\text {rad }} \sim 1 \mu \mathrm{s}\right)$ with $\sigma /\left(k_{\mathrm{B}} T\right)=6$, if the carrier concentration is small.

Second, the enhancement of the capture radius becomes strongly carrier density dependent. Due to the carrier concentration dependence of the mobility and diffusion coefficient (see Table I), the effect decreases with increasing carrier concentration. This effect reduces or even more than compensates the increase of the capture radius with increasing concentration, found for systems without disorder.

Third, at the spatial and time scales considered, the macroscopic diffusion coefficient based model developed in Sec. II is no longer fully adequate, as was already evident from the simulated time dependence of the fraction of quenched excitons (see Fig. 2 and Sec. IV A). The consequences are most clearly visible in Fig. 8(c), for $\sigma /\left(k_{\mathrm{B}} T\right)=6$. For the largest emissive lifetime considered ( $\tau_{\text {rad }}=10^{4} \tau_{\text {rad }, 0} \cong 0.02 \mathrm{~s}$ ), $R_{c \text {,eff }}$ is larger than as expected for very small concentrations, and smaller than as expected for large concentrations. The (small) EPQ loss for low carrier concentrations is for large $\sigma$ and large $\tau_{\text {rad }}$ enhanced due to faster-than-expected short-range diffusion, and the (large) EPQ loss for high concentrations is then somewhat lowered by the occurrence of "protecting" high-energy sites around a small fraction of the excitons (see Sec. IV A).

\section{SUMMARY AND CONCLUSIONS}

In this paper, we have shown that for immediate nearestneighbor (Dexter-type) EPQ in energetically disordered materials, the commonly used description of the steady-state exciton-polaron-quenching rate as the product of a constant rate coefficient $\left(k_{\mathrm{EPQ} \text {,eff }}\right)$, the polaron density, and the exciton density, can be highly inaccurate. From kMC simulations, we find that a description of the EPQ process in terms of an EPQ capture radius can lead to effective values which are significantly (more than one order of magnitude) larger than the value of $\sim 1 \mathrm{~nm}$ which is expected from a nearestneighbor capture process. We furthermore find that the rate coefficient can depend strongly on the polaron concentration. The effect is largest for strongly disordered systems containing emitters with a short emissive lifetime. Finally, the EPQ rate coefficient is found to show a significant electric-field dependence resulting from a "polaron wind" effect. For small and moderate fields, the latter effect is found to be described well using a capture cross-section formalism [Eq. (16)], within which it gives rise to an additional $5 \mathrm{~nm}^{2}$ contribution to the capture radius, equal to the geometrical value.

Our results show that reducing the emission efficiency loss due to the EPQ, realized by replacing the emitter with a material with a reduced emissive lifetime, can be significantly less effective than as expected when assuming a constant effective capture radius. This effect can already be understood in part from the well-known macroscopic diffusion theory of capture processes, within which a contribution of short-range quasi-1D diffusion to the capture site is included. 
However, when considering disordered systems, we find distinct deviations from that theory, related to the very small time and spatial scales involved in the capture process. Such deviations are already observed in the time dependence of the fraction of quenched excitons, shown in Fig. 2. They are also clearly reflected by the explicitly calculated dependence of the steady-state capture radius on the emissive lifetime, the carrier concentration, and the disorder strength, shown in Fig. 8.

We suggest that future exploratory studies of EPQ in any arbitrary material (including, e.g., systems with longer-range EPQ or compositionally mixed host-guest systems) should start with a calculation of quenched fraction curves such as shown in Fig. 2. These curves provide a quick estimate of the efficiency loss due to EPQ, which may be approximated by the fraction of quenched excitons $f_{Q}(\tau)$ at the effective emissive lifetime $\tau$. Furthermore, their precise shape reveals disorder-induced deviations from the EPQ rate coefficient that is expected from macroscopic diffusion theory.

\section{ACKNOWLEDGMENTS}

This work was supported by the Horizon-2020 EU project MOSTOPHOS [Project No. 646259 (R.C., P.A.B.)], the Dutch-German project Modeling of organic light-emitting diodes: from molecule to device [MODEOLED (L.Z.)], and the Dutch nanotechnology program NanoNextNL (H.v.E.). MODEOLED is funded on the Dutch side by the Dutch Technology Foundation STW, the applied science division of NWO, and the Technology Program of the Dutch Ministry of Economic Affairs (Project No. 12200). Part of the work was carried out at the Philips Research Laboratories, Eindhoven, The Netherlands (R.C., L.Z., and H.v.E.).

\section{APPENDIX: SIMULATION DATA}

Tables II-V provide an overview of the kMC simulation results on exciton-polaron quenching at low electric fields, for systems with $\sigma /\left(k_{\mathrm{B}} T\right)=0,2,4$, and 6 , respectively. The simulation data were obtained and analyzed as follows.

As a first step, the (self-)diffusion coefficient $D$ is derived from the mobility, using the classical Einstein equation [Eq. (15)]. The diffusion coefficients obtained are given in Table I in the main text. The electric field for which these simulations were carried out was $F_{\text {sim }}=5 \times 10^{5} \mathrm{~V} / \mathrm{m}$ for systems without disorder $\left[\sigma /\left(k_{\mathrm{B}} T\right)=0\right]$, and $F_{\text {sim }}=10^{7} \mathrm{~V} / \mathrm{m}$ for systems with $\sigma /\left(k_{\mathrm{B}} T\right)=2,4$, and 6 . At these low fields, the estimated field enhancement of the mobility (and diffusion coefficient) is in all cases around $1 \%$ or less. The first simulation result given in Tables II-V is the diffusion length $(D \tau)^{1 / 2}$, with $\tau=0.75 \times \tau_{\text {rad }}$ the effective exciton decay time. A significant enhancement of the effective capture radius due to short-range quasi-1D diffusion is expected when the diffusion length is around $1 \mathrm{~nm}$, or smaller.

Subsequently, the effective EPQ rate coefficient $k_{\mathrm{EPQ} \text {,eff }}$ is obtained from the calculated ratio of quenched and radiatively decayed excitons $f_{Q} / f_{R}$ using Eq. (3). Obtaining this ratio with a high accuracy is most difficult for long exciton lifetimes because the fraction of radiatively decaying excitons is then very small (an effect which is most prominent for high polaron densities) or because the simulation time needed before good
TABLE II. Results of kinetic Monte Carlo simulations of excitonpolaron quenching in organic semiconductors without Gaussian energetic disorder $\left[\sigma /\left(k_{\mathrm{B}} T\right)=0\right]$, carried out under low-field conditions $\left(F_{\text {sim }}=5 \times 10^{5} \mathrm{~V} / \mathrm{m}\right)$. The simulations were performed for various values of the hole concentration $c_{h}$ and the relative radiative decay time $\tau_{\text {rad }} / \tau_{\text {rad }, 0}$, with $\tau_{\text {rad }, 0}=1.84 \mu \mathrm{s}$. The table gives the polaron diffusion length $(D \tau)^{1 / 2}$, the ratio of quenched and radiatively decayed excitons $f_{Q} / f_{R}$, the effective EPQ rate coefficient $k_{\mathrm{EPQ} \text {,eff }}$, and the effective capture radius $R_{c \text {,eff }}$.

\begin{tabular}{llclcc}
\hline \hline & & $\sqrt{D \tau}$ & & $k_{\mathrm{EPQ}, \text { eff }}$ & $R_{c, \text { eff }}$ \\
$c_{h}$ & $\tau_{\text {rad }} / \tau_{\text {rad,0 }}$ & $(\mathrm{nm})$ & $f_{Q} / f_{R}$ & $\left(10^{-18} \mathrm{~m}^{3} \mathrm{~s}^{-1}\right)$ & $(\mathrm{nm})$ \\
\hline $10^{-5}$ & 0.1 & 67.5 & 0.707 & 384 & 0.93 \\
& 0.01 & 21.3 & 0.0715 & 388 & 0.94 \\
& 0.001 & 6.75 & 0.00778 & 423 & 1.03 \\
& $10^{-4}$ & 2.13 & 0.00103 & 560 & 1.36 \\
& $10^{-5}$ & 0.67 & $2.11 \times 10^{-4}$ & 1147 & 2.80 \\
$10^{-4}$ & 0.1 & 67.5 & 7.12 & 387 & 0.94 \\
$10^{-3}$ & 0.1 & 67.5 & 77.7 & 422 & 1.03 \\
& 0.01 & 21.3 & 7.88 & 428 & 1.04 \\
& 0.001 & 6.75 & 0.837 & 455 & 1.11 \\
& $10^{-4}$ & 2.13 & 0.107 & 582 & 1.42 \\
$10^{-2}$ & $10^{-5}$ & 0.67 & 0.00218 & 1185 & 2.89 \\
& 0.1 & 67.5 & $1.04 \times 10^{3}$ & 564 & 1.37 \\
& 0.01 & 21.3 & 94.8 & 515 & 1.25 \\
& 0.001 & 6.75 & 9.80 & 532 & 1.30 \\
& $10^{-4}$ & 2.13 & 1.16 & 630 & 1.54 \\
& $10^{-5}$ & 0.67 & 0.226 & 1228 & 3.00 \\
\hline \hline
\end{tabular}

TABLE III. Results of kinetic Monte Carlo simulations of exciton-polaron quenching in organic semiconductors with a Gaussian disorder strength $\sigma /\left(k_{\mathrm{B}} T\right)=2$, carried out for $F_{\text {sim }}=10^{7} \mathrm{~V} / \mathrm{m}$. The reader is referred to the caption of Table II for an explanation of the quantities included.

\begin{tabular}{|c|c|c|c|c|c|}
\hline$c_{h}$ & $\tau_{\mathrm{rad}} / \tau_{\mathrm{rad}, 0}$ & $\begin{array}{l}\sqrt{D \tau} \\
(\mathrm{nm})\end{array}$ & $f_{Q} / f_{R}$ & $\begin{array}{c}k_{\mathrm{EPQ}, \text { eff }} \\
\left(10^{-18} \mathrm{~m}^{3} \mathrm{~s}^{-1}\right)\end{array}$ & $\begin{array}{l}R_{c, \text { eff }} \\
(\mathrm{nm})\end{array}$ \\
\hline \multirow[t]{6}{*}{$10^{-5}$} & 10 & 212 & 8.14 & 44.2 & 0.93 \\
\hline & 1 & 67.0 & 0.842 & 45.8 & 0.97 \\
\hline & 0.1 & 21.2 & 0.092 & 50.0 & 1.07 \\
\hline & 0.01 & 6.70 & 0.0097 & 52.7 & 1.14 \\
\hline & 0.001 & 2.12 & 0.00116 & 63 & 1.39 \\
\hline & $10^{-4}$ & 0.67 & $2.4 \times 10^{-4}$ & 130 & 3.04 \\
\hline $10^{-4}$ & 1 & 67.2 & 8.30 & 45.1 & 0.94 \\
\hline \multirow[t]{6}{*}{$10^{-3}$} & 10 & 212 & 858 & 46.6 & 0.96 \\
\hline & 1 & 67 & 86.8 & 47.2 & 0.97 \\
\hline & 0.1 & 21 & 8.80 & 47.8 & 0.99 \\
\hline & 0.01 & 6.7 & 0.946 & 51.4 & 1.07 \\
\hline & 0.001 & 2.1 & 0.121 & 65.8 & 1.41 \\
\hline & $10^{-4}$ & 0.67 & 0.025 & 136 & 3.09 \\
\hline \multirow[t]{5}{*}{$10^{-2}$} & 1 & 71.3 & $1.15 \times 10^{3}$ & 62.5 & 1.19 \\
\hline & 0.1 & 22.5 & 119 & 64.7 & 1.24 \\
\hline & 0.01 & 7.13 & 12.1 & 65.8 & 1.26 \\
\hline & 0.001 & 2.25 & 1.44 & 78.3 & 1.53 \\
\hline & $10^{-4}$ & 0.713 & 0.278 & 151 & 3.10 \\
\hline
\end{tabular}


TABLE IV. Results of kinetic Monte Carlo simulations of exciton-polaron quenching in organic semiconductors with a Gaussian disorder strength $\sigma /\left(k_{\mathrm{B}} T\right)=4$, carried out for $F_{\text {sim }}=10^{7} \mathrm{~V} / \mathrm{m}$. The reader is referred to the caption of Table II for an explanation of the quantities included.

\begin{tabular}{|c|c|c|c|c|c|}
\hline$c_{h}$ & $\tau_{\mathrm{rad}} / \tau_{\mathrm{rad}, 0}$ & $\begin{array}{l}\sqrt{D \tau} \\
(\mathrm{nm})\end{array}$ & $f_{Q} / f_{R}$ & $\begin{array}{c}k_{\mathrm{EPQ}, \mathrm{eff}} \\
\left(10^{-18} \mathrm{~m}^{3} \mathrm{~s}^{-1}\right)\end{array}$ & $\begin{array}{l}R_{c, \text { eff }} \\
(\mathrm{nm})\end{array}$ \\
\hline \multirow[t]{6}{*}{$10^{-5}$} & 100 & 49.7 & 0.534 & 0.290 & 1.13 \\
\hline & 10 & 15.7 & 0.059 & 0.321 & 1.26 \\
\hline & 1 & 4.97 & 0.0063 & 0.342 & 1.36 \\
\hline & 0.1 & 1.57 & $8.18 \times 10^{-4}$ & 0.444 & 1.81 \\
\hline & 0.01 & 0.497 & $1.87 \times 10^{-4}$ & 1.02 & 4.34 \\
\hline & 0.001 & 0.157 & $9.60 \times 10^{-5}$ & 5.21 & 22.9 \\
\hline \multirow[t]{6}{*}{$10^{-4}$} & 100 & 57.5 & 5.92 & 0.322 & 0.91 \\
\hline & 10 & 18.2 & 0.667 & 0.362 & 1.04 \\
\hline & 1 & 5.75 & 0.0817 & 0.444 & 1.32 \\
\hline & 0.1 & 1.82 & 0.00987 & 0.536 & 1.62 \\
\hline & 0.01 & 0.575 & 0.00225 & 1.22 & 3.90 \\
\hline & 0.001 & 0.182 & 0.00114 & 6.20 & 20.4 \\
\hline \multirow[t]{6}{*}{$10^{-3}$} & 100 & 77.1 & 105 & 0.581 & 0.90 \\
\hline & 10 & 24.4 & 11.1 & 0.603 & 0.96 \\
\hline & 1 & 7.71 & 1.29 & 0.701 & 1.14 \\
\hline & 0.1 & 2.44 & 0.170 & 0.924 & 1.55 \\
\hline & 0.01 & 0.771 & 0.032 & 1.74 & 3.06 \\
\hline & 0.001 & 0.244 & 0.013 & 7.06 & 12.9 \\
\hline \multirow[t]{5}{*}{$10^{-2}$} & 10 & 40.5 & 340 & 1.85 & 1.08 \\
\hline & 1 & 12.8 & 34.9 & 1.90 & 1.11 \\
\hline & 0.1 & 4.05 & 4.21 & 2.29 & 1.38 \\
\hline & 0.01 & 1.28 & 0.698 & 3.59 & 2.38 \\
\hline & 0.001 & 0.405 & 0.199 & 10.8 & 7.08 \\
\hline
\end{tabular}

time averaging is obtained becomes very large (an effect which is most prominent for low polaron densities). These considerations have affected the range of radiative lifetimes for which the results are displayed. The estimated numerical accuracy of the $f_{Q} / f_{R}$ ratios is approximately $5 \%$ or better.
TABLE V. Results of kinetic Monte Carlo simulations of excitonpolaron quenching in organic semiconductors with a Gaussian disorder strength $\sigma /\left(k_{\mathrm{B}} T\right)=6$, carried out for $F_{\mathrm{sim}}=10^{7} \mathrm{~V} / \mathrm{m}$. The reader is referred to the caption of Table II for an explanation of the quantities included.

\begin{tabular}{|c|c|c|c|c|c|}
\hline$c_{h}$ & $\tau_{\mathrm{rad}} / \tau_{\mathrm{rad}, 0}$ & $\begin{array}{l}\sqrt{D \tau} \\
(\mathrm{nm})\end{array}$ & $f_{Q} / f_{R}$ & $\begin{array}{c}k_{\mathrm{EPQ}, \mathrm{eff}} \\
\left(10^{-18} \mathrm{~m}^{3} \mathrm{~s}^{-1}\right)\end{array}$ & $\begin{array}{l}R_{c, \text { eff }} \\
(\mathrm{nm})\end{array}$ \\
\hline \multirow[t]{6}{*}{$10^{-5}$} & 10000 & 16.5 & 0.065 & $3.53 \times 10^{-4}$ & 1.32 \\
\hline & 1000 & 5.27 & 0.0067 & $3.64 \times 10^{-4}$ & 1.37 \\
\hline & 100 & 1.65 & $7.9 \times 10^{-4}$ & $4.29 \times 10^{-4}$ & 1.64 \\
\hline & 10 & 0.527 & $1.6 \times 10^{-4}$ & $8.70 \times 10^{-4}$ & 3.49 \\
\hline & 1 & 0.165 & $6.9 \times 10^{-5}$ & 0.00375 & 15.5 \\
\hline & 0.1 & 0.0527 & $6.0 \times 10^{-5}$ & 0.0326 & 136 \\
\hline \multirow[t]{2}{*}{$10^{-4}$} & 10000 & 29.3 & 1.67 & $9.08 \times 10^{-4}$ & 1.00 \\
\hline & 1 & 0.293 & 0.00153 & 0.00831 & 10.5 \\
\hline \multirow[t]{6}{*}{$10^{-3}$} & 10000 & 65.7 & 64.1 & 0.0035 & 0.74 \\
\hline & 1000 & 20.8 & 7.46 & 0.0041 & 0.89 \\
\hline & 100 & 6.57 & 1.07 & 0.0058 & 1.34 \\
\hline & 10 & 2.08 & 0.151 & 0.0082 & 1.95 \\
\hline & 1 & 0.657 & 0.0302 & 0.0164 & 4.06 \\
\hline & 0.1 & 0.208 & 0.0140 & 0.0761 & 19.4 \\
\hline \multirow[t]{5}{*}{$10^{-2}$} & 1000 & 66.2 & 645 & 0.0353 & 0.72 \\
\hline & 100 & 20.9 & 78.5 & 0.0427 & 0.91 \\
\hline & 10 & 6.62 & 9.83 & 0.0534 & 1.17 \\
\hline & 1 & 2.09 & 1.49 & 0.0811 & 1.86 \\
\hline & 0.1 & 0.662 & 0.365 & 0.198 & 4.78 \\
\hline
\end{tabular}

As a final step, the methodology developed in Sec. II C for extrapolating the simulation data to zero electric field is used to derive the effective capture radius $R_{c, \text { eff }}$, using Eq. (18). This approach corrects for an $A_{c, \infty}^{\prime}=5 \mathrm{~nm}^{2}$ polaron wind contribution to the effective capture cross section. For the electric fields used, the correction is negligible when $\sigma /\left(k_{\mathrm{B}} T\right)=0$ and $0.155 \mathrm{~nm}$ for the disordered systems considered.
[1] C. Murawski, K. Leo, and M. C. Gather, Adv. Mater. 25, 6801 (2013).

[2] J. M. Hodgkiss, S. Albert-Seifried, A. Rao, A. J. Barker, A. R. Campbell, R. A. Marsh, and R. H. Friend, Adv. Funct. Mater. 22, 1567 (2012).

[3] I. A. Howard, J. M. Hodgkiss, X. Zhang, K. R. Kirov, H. A. Bronstein, C. K. Williams, R. H. Friend, S. Westenhoff, and N. C. Greenham, J. Am. Chem. Soc. 132, 328 (2009).

[4] B. Verreet, A. Bhoolokam, A. Brigeman, R. Dhanker, D. Cheyns, P. Heremans, A. Stesmans, N. C. Giebink, and B. P. Rand, Phys. Rev. B 90, 115304 (2014).

[5] E. J. W. List, C.-H. Kim, A. K. Naik, U. Scherf, G. Leising, W. Graupner, and J. Shinar, Phys. Rev. B 64, 155204 (2001).

[6] N. H. Hansen, C. Wunderlich, A. K. Topczak, E. Rohwer, H. Schwoerer, and J. Pflaum, Phys. Rev. B 87, 241202 (2013).

[7] M. A. Baldo, C. Adachi, and S. R. Forrest, Phys. Rev. B 62, 10967 (2000).
[8] S. Reineke, K. Walzer, and K. Leo, Phys. Rev. B 75, 125328 (2007).

[9] D. Hertel and K. Meerholz, J. Phys. Chem. B 111, 12075 (2007).

[10] J. Kalinowski, W. Stampor, J. Szmytkowski, D. Virgili, M. Cocchi, V. Fattori, and C. Sabatini, Phys. Rev. B 74, 085316 (2006).

[11] N. C. Erickson and R. J. Holmes, Adv. Funct. Mater. 24, 6074 (2014).

[12] S. Oyama, H. Sakai, and H. Murata, Jpn. J. Appl. Phys. 55, 03DD13 (2016).

[13] K. W. Hershey and R. J. Holmes, J. Appl. Phys. 120, 195501 (2016).

[14] M. von Smoluchowski, Z. Phys. Chem. 92, 129 (1917).

[15] S. Chandrasekhar, Rev. Mod. Phys. 15, 1 (1943).

[16] M. Pope and C. E. Swenberg, Electronic Processes in Organic Crystals and Polymers (Oxford University Press, Oxford, 1999). 
[17] B. Valeur and M. N. Berberan-Santos, Molecular Fluorescence: Principles and Applications (Wiley, Hoboken, NJ, 2012).

[18] J. J. M. van der Holst, F. W. A. van Oost, R. Coehoorn, and P. A. Bobbert, Phys. Rev. B 80, 235202 (2009).

[19] A. Massé, R. Coehoorn, and P. A. Bobbert, Phys. Rev. Lett. 113, 116604 (2014).

[20] Y. Shen and N. C. Giebink, Phys. Rev. Appl. 4, 054017 (2015).

[21] H. van Eersel, P. A. Bobbert, R. A. J. Janssen, and R. Coehoorn, Appl. Phys. Lett. 105, 143303 (2014).

[22] H. van Eersel, P. A. Bobbert, R. A. J. Janssen, and R. Coehoorn, J. Appl. Phys. 119, 163102 (2016).

[23] M. Mesta, H. van Eersel, R. Coehoorn, and P. A. Bobbert, Appl. Phys. Lett. 108, 133301 (2016).

[24] The BUMBLEBEE kinetic Monte Carlo software is provided by Simbeyond B.V. (http://simbeyond.com).

[25] R. Coehoorn, H. van Eersel, P. A. Bobbert, and R. A. J. Janssen, Adv. Funct. Mater. 25, 2024 (2015).

[26] H. van Eersel, P. A. Bobbert, and R. Coehoorn, J. Appl. Phys. 117, 115502 (2015).

[27] L. Zhang, H. van Eersel, P. A. Bobbert, and R. Coehoorn, Chem. Phys. Lett. 652, 142 (2016).
[28] A. Miller and E. Abrahams, Phys. Rev. 120, 745 (1960).

[29] M. Mesta, M. Carvelli, R. J. de Vries, H. van Eersel, J. J. M. van der Holst, M. Schober, M. Furno, B. Lüssem, K. Leo, P. Loebl, R. Coehoorn, and P. A. Bobbert, Nat. Mater. 12, 652 (2013).

[30] R. A. Marcus, Rev. Mod. Phys. 65, 599 (1993).

[31] J. Cottaar, L. J. A. Koster, R. Coehoorn, and P. A. Bobbert, Phys. Rev. Lett. 107, 136601 (2011).

[32] J. Zhou, Y. C. Zhou, J. M. Zhao, C. Q. Wu, X. M. Ding, and X. Y. Hou, Phys. Rev. B 75, 153201 (2007).

[33] W. C. Germs, J. J. M. van der Holst, S. L. M. van Mensfoort, P. A. Bobbert, and R. Coehoorn, Phys. Rev. B 84, 165210 (2011).

[34] F. Jansson, A. V. Nenashev, S. D. Baranovskii, F. Gebhard, and R. Österbacka, Ann. Phys. (NY) 18, 856 (2009).

[35] A. V. Nenashev, F. Jansson, S. D. Baranovskii, R. Österbacka, A. V. Dvurechenskii, and F. Gebhard, Phys. Rev. B 81, 115204 (2010).

[36] Y. Roichman and N. Tessler, Appl. Phys. Lett. 80, 1948 (2002).

[37] J. C. Dyre and T. Schrøder, Rev. Mod. Phys. 72, 873 (2000).

[38] W. F. Pasveer, J. Cottaar, C. Tanase, R. Coehoorn, P. A. Bobbert, P. W. M. Blom, D. M. de Leeuw, and M. A. J. Michels, Phys. Rev. Lett. 94, 206601 (2005). 